\title{
PRACTICING WITH GRADUAL INCREASES IN CONTEXTUAL INTERFERENCE ENHANCES VISUOMOTOR LEARNING
}

\author{
Jared M. Porter and Trey Beckerman \\ Department of Kinesiology, Southern Illinois University, Carbondale, Illinois, USA
}

Original scientific paper

UDC: $796.012: 519.2$

\begin{abstract}
:
The purpose of this study was to determine if practicing with gradual increases in contextual interference (CI) facilitated the learning of a continuous motor skill that required visuomotor tracking. We hypothesized the group that practiced with increasing amounts of CI would perform significantly better on a retention and transfer test compared to participants that practiced with blocked and random scheduling. A total of 78 participants were randomly assigned to one of three groups (i.e. Blocked, Increasing, Random). The level of CI was modified by varying the number of revolutions per minute (RPM) on a rotary pursuit tracker. Following the acquisition phase, participants returned after a 24-hour period and completed the 12-trial retention and transfer test. The results of statistical analysis indicated that all three groups improved their performance during practice. The posttest analysis indicated the Increasing group was better than the Blocked and Random groups on the retention and transfer test.
\end{abstract}

Key words: visual, tracking, skill acquisition

\section{Introduction}

Many factors contribute to the skill acquisition process such as how much contextual interference the learner encounters when practicing multiple skills. Contextual interference (CI) is defined as the interference in learning and performance that occurs when practicing a task in the context of other tasks (Schmidt \& Lee, 2011). Manipulating the order of practice trials can change the amount of $\mathrm{CI}$ that an individual is faced with when practicing two or more tasks (Magill \& Hall, 1990). When deciding the correct way to implement CI into a practice schedule, it is important to understand that $\mathrm{CI}$ exists on a continuum, beginning with blocked scheduling (i.e. low CI), and ending with random scheduling (i.e. high CI). A blocked schedule consists of multiple same task repetitions prior to moving on to another task variation. In contrast, a random schedule is characterized by practicing multiple tasks in a changing unpredictable order. Past studies have shown that experiencing higher levels of CI during practice may show minimal improvement in motor performance. However, it often leads to superior performance on post-practice transfer and retention tests when compared to the conditions that practiced with lower levels of CI (Lee \& Magill, 1983; Shea \& Morgan, 1979). This phenomenon is often referred to as the contextual interference effect (Brady, 1998; Magill \& Hall, 1990; Magill, 2011). It is worth noting that practicing with moderate amounts of CI (e.g. serial scheduling) has also been shown to benefit motor skill learning (Landin \& Hebert, 1997). Additionally, some authors have suggested that practicing with a moderate amount of contextual interference results in learning effects that are similar to practicing with high amounts of contextual interference in a random schedule (Lee \& Magill, 1983).

Studies examining the $\mathrm{CI}$ effect have commonly used fixed amounts of CI during practice to investigate how CI interacts with the motor learning process. In attempting to explain the absence of the CI effect in certain learners, Landin and Herbert (1997) suggested that the skill level of the learner in a specific task was the source of the conflicting results because the more experienced learners would benefit more from high CI (e.g. random scheduling), while novice learners would benefit more from low CI (e.g. blocked scheduling). However, if the level of CI is too low, learners can experience what Magill (2011) refers to as "contextual dependency". Contextual dependency occurs when the learner develops a dependency on the context in which practice occurs. This leads to decreased performance during a transfer test when the context of the performance changes (Kimbrough, Wright \& Shea, 2001; Shea \& Wright, 1995). In contrast, 
if the amount of CI experienced in practice is too high, then the learning process may be compromised because the learner is overwhelmed by the constantly changing context of practice (Guadagnoli, Holcomb, \& Weber, 1999). Empirical findings such as these suggest the decision to introduce a particular amount of CI in practice should be based on the skill level of the learner.

The concept of introducing varying amount of $\mathrm{CI}$ in practice based on a learner's skill level, led Porter and Magill (2010) to investigate the benefits of practicing with gradual increases in CI. In a set of experiments Porter and Magill (2010) had novices practice golf putting (Experiment 1) and basketball-related passes (Experiment 2) with blocked, random, or "increasing" amounts of CI. Participants in the Increasing group practiced the first one-third of practice with blocked scheduling (i.e. low CI). The middle phase of practice consisted of serial scheduling (i.e. moderate CI). This group of participants then concluded practice with a random arrangement of practice trials (i.e. high CI). Results of these experiments demonstrated learning benefits for participants practicing with systematic increases in CI, compared to the participants practicing with a traditional fixed blocked and random scheduling. Additional support for this form of practice was later reported for moderately skilled participants (Porter \& Saemi, 2010) and elementary school-aged children learning overarm throwing (Porter, Saemi, Varzaneh, Zarghami, \& Shafinia, 2011; Saemi, Porter, Ghotbi-Varzaneh, Zarghami, \& Shafinia, 2012).

Porter and Magill (2010) used the challengepoint hypothesis (Guadagnoli \& Lee, 2004) and Bjork's (1994, 1999) "desirable difficulties" model to explain the benefits of practicing with gradual increases in CI. Desirable difficulties (Bjork, 1994, 1999) are certain practice conditions that cause the learner to fully engage in the task at hand and put substantial cognitive effort into the learning process. Presumably, this increased cognitive engagement facilitates transfer and retention test performances. Contextual interference can be a vehicle for incorporating desirable difficulties into a practice schedule (Porter \& Magill, 2010). The challengepoint hypothesis (Guadagnoli \& Lee, 2004) extends the desirable difficulties concept by suggesting that the difficulty in practice conditions is a function of the relationship between nominal task difficulty and functional task difficulty. Nominal task difficulty refers to the constant level of difficulty of a given task, independent of the performer's skill level. By point of comparison, functional task difficulty refers to the level of difficulty of a given task relative to the performer's skill level or performance situation. When a learner's performance of a task improves, the level of functional task difficulty consequently decreases. In order to make further improvements in performance as a learner's skill level advances, the nominal task difficulty must increase to offset the decrease in functional task difficulty. Porter and Magill (2010) proposed the desired constant state of challenge/difficulty can be accomplished by systematically increasing the amount of CI in practice as learners simultaneously improve their skill level and their ability to process critical information. Through Porter and Magill's (2010) parallel development hypothesis, it is suggested that as the skill level and information processing abilities are improving, the practice schedule should evolve with increasing amounts of difficulty to constantly challenge the learner at more optimal levels. Practicing with gradual increases in CI appears to be one way to create this optimal practice environment (Porter \& Magill, 2010; Porter \& Saemi, 2010; Porter, et al., 2011).

The benefits of practicing with gradual increases in CI have been replicated using a variety of tasks (e.g. golf putting, basketball passing, overarm throwing) in diverse populations (e.g. children, novice adults, moderately skilled adults). However, questions still remain about the appropriateness of this form of practice. Thus far, all reported investigations of an increasing practice schedule have used discrete projectile-based motor skills (e.g. golf putting, throwing). By their nature, these types of tasks are very short in duration (i.e. discrete) and require the participants to preplan their movements prior to the initiation of the task. In addition, projectile-based skills require the learners to plan their movement in terms of their desired outcomes, such as hitting the center of the target located some distance from them. To better understand potential limitations and benefits of an increasing practice schedule, there is a need to investigate this phenomenon using more demanding tasks, such as continuous skills that require visuomotor tracking. Compared to discrete skills, continuous skills are longer in duration and encourage the learner to use inherent feedback to make needed on-line modifications to improve performance (Magill, 2011).

The purpose of this study was to examine the generalizability of the findings presented by Porter and Magill (2010). Specifically, we investigated if practicing with gradual increases in CI also facilitated the learning of a novel continuous motor skill that required visual and motor tracking. We compared this form of practicing to the traditional blocked and random scheduling. We hypothesized that participants practicing with systematic increases in CI would show performance improvements during practice. We also predicted that the participants practiced with increasing amounts of CI would perform significantly better on a retention and transfer test compared to the participants that practiced with blocked and random scheduling. Such a finding would indicate that the benefits of 
an increasing practice schedule are not limited to discrete preplanned tasks.

\section{Method}

\section{Participants}

Participants $(\mathrm{N}=78)$ were college-aged students enrolled in various courses at a university in the United States of America. There were equal numbers of male and female participants in each of the three experimental conditions. All methods were approved by a University's Institutional Review Board. All participants were naive to the purpose of the study, and signed an informed consent prior to their participation in the present experiment.

\section{Apparatus and task}

Participants practiced a rotary pursuit tracking task. The task required participants to sit and track a rotating light at speeds of 20,30 , and 40 revolutions per minute (RPM). The rotating light had a surface area of $2 \times 2 \mathrm{~cm}$ and traveled in a clockwise circular direction; the circle template had a diameter of $30 \mathrm{~cm}$. A square template was used during the transfer test; the square template measured $20 \times 20$ $\mathrm{cm}$. Each participant held a wand in their dominant hand; the wand contained a light-sensitive photocell in its tip. The goal of each participant was to use the handheld wand to track the rotating light throughout each trial. Specifically, participants were instructed to keep the tip of the wand in constant contact with the rotating light. The device measured the time the wand was in contact with the light, thus total time on target served as the dependent measure in the present experiment. Participants were not provided any feedback about their performances.

\section{Procedure}

Once each participant read and signed the consent form, they were randomly placed into one of three practice groups: Blocked, Increasing, or Random. On day one, each participant performed 36 practice trials of the rotary pursuit task using a circle template. The duration of each trial was 10 seconds, with a five second interval between trials. The Blocked group performed 12 trials of each RPM speed in counterbalanced blocks. Participants assigned to the Increasing CI group followed a practice schedule that introduced the learner to gradual increases in CI. For example, their first 12 trials followed a counterbalanced blocked schedule (i.e. low CI) with four trials at 20 RPM, four trials at 30 RPM, and four trials at 40 RPM in a repeated fashion. The next 12 trials of the Increasing schedule were performed following a serial schedule (i.e. moderate CI) where the pattern of one trial at 20 RPM was followed by one trial at 30 RPM and then one trial at $40 \mathrm{RPM}$. This pattern was repeated four times for a total of 12 trials. The final 12 trials of the Increasing CI group were performed in a random (i.e. high CI) order, with the constraint that each speed was practiced four times and no same speed trials were performed for more than two consecutive trials. The Random CI group performed 36 trials in a random order with no RPM occurring more than twice in a row, and each RPM being performed 12 times. Counterbalancing was used throughout all three experimental conditions to control for possible order effects.

After a 24-hour period, participants returned for a retention and transfer test. In both of these tests, participants followed a novel mini-blocked schedule of two trials at 20 RPM, two trials at 30 RPM, and two trials at 40 RPM. This sequence was performed twice in both the retention test and transfer test for a total of 12 trials for each test. The retention test procedure was done with the same circle template utilized during practice, and the transfer test used a square template.

\section{Results}

\section{Practice}

Practice trial performances were analyzed using a three (Condition) $\times 12$ (Trial Block) analysis of variance (ANOVA) with repeated measures on the last factor. Each trial block consisted of the average score for three trials. Specifically, trial block one was comprised of the average score (i.e. time on target) for the first attempt of each of the three rotary pursuit speeds (i.e. 20, 30, 40 RPM). The second trial block was the average score for the second attempt at each speed, this calculation continued until the $12^{\text {th }}$ trial of each speed. The analysis revealed a main effect for Condition $F(2,75)=3.65, \mathrm{p}=.031$. The ANOVA also indicated there was a main effect for Trial Block $F(11$, $825)=4.73, \mathrm{p}<.001$. There was no Condition $\times$ Trial Block interaction $F(2,75) \mathrm{p}>.05$. A Least Significant Differences (LSD) follow-up analysis of Conditions further revealed that the Increasing and Block schedules were significantly better than the Random schedule throughout the acquisition phase. Additionally, a follow-up analysis on the Trial Block main effect indicated all three practice schedules improved their performance during practice (see Figure 1).

\section{Retention and transfer tests}

Retention and transfer test data were analyzed with separate one-way ANOVAs. The analysis of the retention test (presented in Figure 2) indicated a main effect for condition $F(2,75)=12.47, \mathrm{p}<.001$. Follow-up analysis (LSD) further revealed that the Random ( $\mathrm{M}=5.54, \mathrm{SD}=0.52 \mathrm{~s})$ and Increasing $(\mathrm{M}=6.04, \mathrm{SD}=0.65 \mathrm{~s})$ groups were significantly 


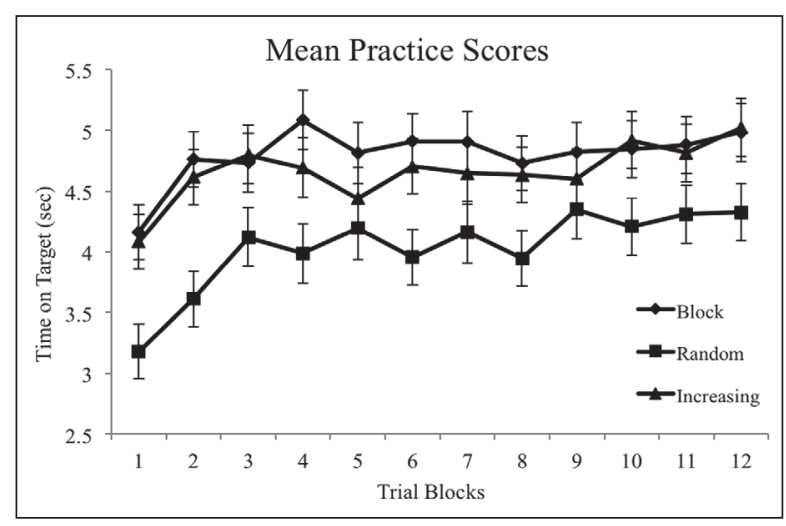

Figure 1. Mean practice scores and standard deviations for the Block, Random, and Increasing groups across the 12 trial blocks of acquisition. Each trial block represents the average score for three trials (i.e. one from each speed).

better than the Block $(\mathrm{M}=5.11, \mathrm{SD}=0.81 \mathrm{~s})$ group. This analysis also determined that the Increasing group performed better than the Random group on the retention test. Similarly, the analysis of transfer test (presented in Figure 3) performances indicated a condition main effect $F(2,75)=11.23, \mathrm{p}<.001$. Consistent with the retention test performance, post-hoc analysis indicated that the Increasing $(\mathrm{M}=4.32, \mathrm{SD}=.73 \mathrm{~s})$ group was significantly better

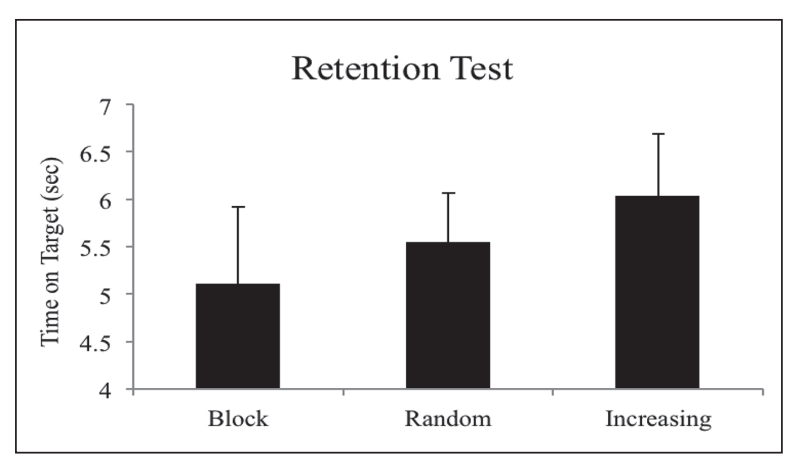

Figure 2. Mean practice score and standard deviation for the Block, Random and Increasing groups during the retention test. Results revealed that all three groups were significantly different from each other.

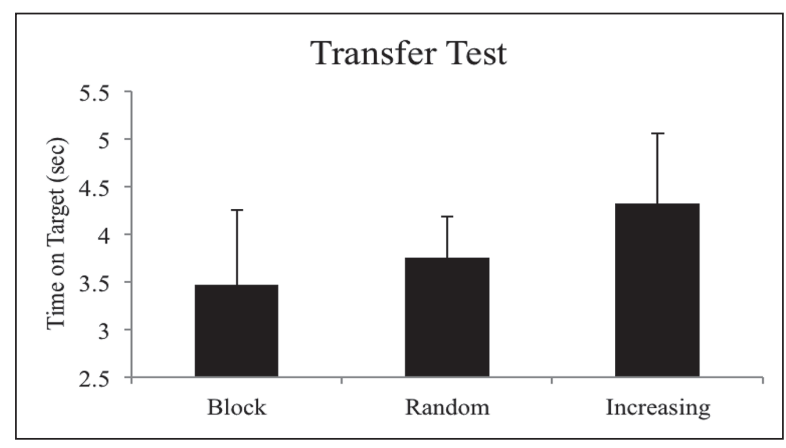

Figure 3. Mean practice score and standard deviation for the Block, Random and Increasing groups during the transfer test. Results revealed that the Increasing group was significantly different from both the Block and Random groups; however, the Block and Random groups were not significantly different. on the transfer test compared to the Block $(\mathrm{M}=3.46$, $\mathrm{SD}=.78 \mathrm{~s})$ and Random $(\mathrm{M}=3.76, \mathrm{SD}=.43 \mathrm{~s})$ schedules. The transfer test follow-up analysis also revealed that the Block and Random groups were not significantly different from each other.

\section{Discussion and conclusions}

Many researchers have investigated the effects of $\mathrm{CI}$ on the ability to learn a novel skill, but most have looked at fixed amounts of CI throughout practice (Brady, 1998). Magill and Hall (1990) suggested future research should investigate alternative practice schedules containing varied amounts of $\mathrm{CI}$ to help explain conflicting results between some CI studies. The purpose of the current study was to test the prediction that practicing with systematic increases in CI would benefit the learning of a continuous visuomotor tracking task. We hypothesized novices practicing with gradual increases in CI would show improved performance during practice. In addition, we predicted that the group practicing with gradual increases in CI would perform significantly better on a retention and transfer test compared to the participants that practiced with traditional blocked and random scheduling. The results of the present study are in line with our predictions; moreover, these findings are consistent with previous research (e.g. Porter \& Magill, 2010; Porter \& Saemi, 2010). The results of the present experiment are meaningful because they provide an initial demonstration that the benefits of scheduling practice with increasing amounts of CI facilitates the learning of a continuous task requiring visual and motor tracking.

Porter and Magill (2010) offer a hypothesized explanation as to why scheduling practice with gradual increases in CI is beneficial for novices. They suggested the amount of information an individual can process is limited; however, information processing ability can be made more efficient through effective practice. The concept of improving information processing ability through practice is not new, and has been explored in previous CI research (see Aloupis, Guadagnoli, \& Kohl, 1995). In addition to research based on information processing theory, the theorized explanation offered by Porter and Magill was also grounded in findings from the stages of learning literature. Specifically, Gentile (1972) suggests when learners are in the early stages of acquiring a motor skill, they need repeated trials to correct movementrelated errors, explore new movement patterns, and determine a way to successfully achieve the action goal. However, continued blocked practice depresses motor learning by creating a context dependency (Magill, 2011). As a result, a practical conundrum is created in the design of effective practice. Meaning that repeated trials are valuable in the early learning process (Gentile, 1972), but 
too much repetition of the same task can result in a contextual dependency which depresses learning (Magill, 2011). Based on the present study's findings, it appears that offering initial blocked practice and then transitioning to serial, and later random scheduling is an effective way to take advantage of early repeated practice trials while not allowing the negative effects of contextual dependency to settle in and harm the acquisition of the practiced tasks.

Not only is it important to neutralize the effects of contextual dependency, it is also imperative that as the learner's ability to process information improves, the amount of CI is increased so that the learner is constantly being challenged at an appropriate level (Porter \& Magill, 2010). This gradual increase in CI may be explained by Bjork's (1994, 1999) description of "desirable difficulties" and the "challenge point hypothesis" (Guadagnoli \& Lee, 2004). The results of the current study support the prediction that increasing $\mathrm{CI}$ as a learner becomes more skilled during practice will lead to enhancements in post-test performance. Seemingly, practicing with gradual increase in CI allows the learners to adjust their own movements while practicing the skill, which results in the development of an efficient movement pattern that will lead them to success. As the CI increases during practice, the learner is constantly challenged at a level that will lead to improved performance when compared to practicing with fixed levels of CI (e.g. blocked or random scheduling).

Consistent with the aforementioned perspectives, we propose that participants assigned to the Increasing group in the present experiment developed a basic movement pattern while completing trials in the initially less challenging and consequently less difficult blocked schedule. This allowed the novices to explore various movement patterns and strategies to gain initial success at the task. However, as the schedule became progressively more challenging by progressing to serial and later random scheduling, the learners were able to manage the difficulties of the elevated CI because of more efficient information processing abilities and a more evolved motor program. It appears that one of the key benefits of an increasing practice schedule is the encouragement of the simultaneous (i.e. parallel) development between information processing abilities and motor program development. As a result, the parallel development between movements and processing ability is more fully developed when the learning environment evolves (as was the case in the Increasing group) to meet the changing processing and motor ability characteristics of the learner. Moreover, the findings of the present study support the conclusion that a practice schedule that has a static amount of CI, as was the case with the Blocked and Random groups, created a less optimal learning environment.
The findings of the current experiment provide evidence that the learning benefits of practicing with systematic increases in CI are not limited to preplanned discrete motor skills, but are also observed within continuous skills that require the learner to monitor and modify their coordination pattern while executing the task. This finding is meaningful not only because it demonstrates the generalizability of findings reported in previous studies (e.g. Porter \& Magill, 2010; Porter \& Saemi, 2010), but also because it demonstrates that novices learning a motor skill that requires continuous monitoring of motor behavior experience superior learning effects when the practice schedule offers progressively elevated amounts of CI. Additionally, this study makes a valuable contribution to the existing body of CI literature because very few studies have examined the CI effect when practicing continuous motor skills (e.g. Smith, 2002).

Besides the learning benefits reported above, it is worth pointing out that the typical CI effect was partially observed in this study, meaning that the Blocked group was better than the Random group during practice, yet the Random group was superior to the Blocked group on the retention test. However, the Blocked and Random groups were not significantly different on the transfer test. This latter finding is not consistent with the traditional CI effect. Additionally, a more meaningful observation is that the Increasing group was significantly better than the Blocked and Random groups on the transfer test. This is a noteworthy observation because previous authors have reported that a transfer test is a more sensitive measure of learning relative to a retention test (Chiviacowsky \& Wulf, 2002; Lai \& Shea, 1998; Wulf \& Lee, 1993). This finding supports the conclusion that practicing with gradual increases in CI is an effective form of practice to facilitate motor skill acquisition.

Future studies should continue to focus on practice schedules constructed with systematic increases in CI. Such research is needed to fully investigate the hypothesis suggesting that the benefits reported here are the result of the optimization between the parallel development of information processing ability and motor skill improvement. This future research is needed to more fully understand the mechanisms that contribute to the interaction of contextual interference and practice schedule design. Pursuing these future lines of investigation will provide answers to the questions that remain about the effectiveness of this alternative form of practice. The pursuit of these questions will make small contributions to the large body of literature striving to bring understanding to the motor learning process.

The findings presented here not only make a theoretical contribution to the existing body of CI literature, there is also a practical relevance to the 
results of this study. Specifically, practitioners can use the guidelines outlined above to better design practice environments for novice learners. For example, based on the results of this experiment, a coach who works with athletes learning a continuous skill (e.g. swimming, cycling, rowing) should begin practice by allowing the individual athletes to practice repeated attempts (i.e. blocked practice) of the prescribed skill. After the athlete has been allowed to repeatedly practice the task, the coach should then progress the athlete into a non- repeating (i.e. serial) form of practice which induces a moderate amount of CI into the learning environment. Finally, the coach should conclude the practice session with random scheduling to optimize motor learning effects. Similar practice methods could easily be adopted by other practitioners such as physical therapists, physical education teachers, or military drill instructors. Utilizing such practices would likely result in enhancements in motor learning.

\section{References}

Aloupis, C.H., Guadagnoli, M.A., \& Kohl, R.M. (1995). Manipulation of task switches during acquisition: A test of traditional contextual interference hypotheses. Journal of Human Movement Studies, 29, 171-180.

Bjork, R.A. (1994). Memory and metamory considerations in the training of human beings. In J. Metcalfe \& A. Shimamura (Eds.), Metacognition: Knowledge about knowing (pp. 185-205). Cambridge, MA: MIT Press.

Bjork, R.A. (1999). Assessing our own competence: Heuristics and illusions. In D. Gopher \& A. Koriat (Eds.), Attention and performance XVII. Cognitive regulation of performance: Interaction of theory and application ( $\mathrm{pp} .435-459)$. Cambridge, MA: MIT Press.

Brady, F. (1998). A theoretical and empirical review of the contextual interference effect and the learning of motor skills. Quest, 50, 266-293.

Chiviacowsky, S., \& Wulf, G. (2002). Self-controlled feedback: Does it enhance learning because performers get feedback when they need it? Research Quarterly for Exercise and Sport, 73, 408-415.

Gentile, A.M., (1972). A working model of skill acquisition with application to teaching. Quest, 17, 3-23.

Guadagnoli, M.A., Holcomb, W.R., \& Weber, T.J. (1999). The relationship between contextual interference effects and performer expertise on the learning of a putting task. Journal of Human Movement Studies, 37, 19-36.

Guadagnoli, M.A., \& Lee, T.D. (2004). Challenge point: A framework for conceptualizing the effects of various practice conditions in motor learning. Journal of Motor Behavior, 36, 212-224.

Herbert, E.P., Landin, D., \& Solomon, M.A. (1996). Practice schedule effects on the performance and learning of lowand high-skilled students: An applied study. Research Quarterly for Exercise and Sport, 67, 52-58.

Kimbrough, S.K., Wright, D.L., \& Shea, C.H. (2001). Reducing the saliency of intentional stimuli results in greater contextual-dependent performance. Memory, 9, 133-143.

Lai, Q., \& Shea, C.H. (1998). Generalized motor program (GMP) learning: Effects of reduced frequency of knowledge of results and practice variability. Journal of Motor Behavior, 30, 51-59.

Landin, D., \& Herbert, E.P. (1997). A comparison of three practice schedules along the contextual interference continuum. Research Quarterly for Exercise and Sport, 68 357-361.

Lee, T.D., \& Magill, R.A., (1983). A locus of contextual interference in motor skill acquisition. Journal of Experimental Psychology: Learning, Memory, \& Cognition, 9, 730-746.

Magill, R.A. (2011). Motor learning and control: Concepts and applications ( ${ }^{\text {th }}$ ed.). New York, NY: McGraw-Hill.

Magill, R.A., \& Hall, K.G. (1990). A review of the contextual interference effects in skill acquisition. Human Movement Science, 9, 241-289.

Porter, J.M., \& Magill, R.A. (2010). Systematically increasing contextual interference is beneficial for learning sport skills. Journal of Sports Sciences, 28, 1277-1285.

Porter, J.M., \& Saemi, E. (2010). Moderately skilled learners benefit by practicing with systematic increases in contextual interference. International Journal of Coaching Science, 4, 61-71.

Porter, J.M., Saemi, E., Varzaneh, A., Zarghami, M., \& Shafinia, P. (2011). Systematically increasing contextual interference during practice is beneficial for children learning a fundamental motor skill. Journal of Sport and Exercise Psychology, 33, S101.

Saemi, E., Porter, J.M., Ghotbi-Varzaneh, A., Zarghami, M., \& Shafinia, P. (2012). Practicing along the contextual interference continuum: A comparison of three practice schedules in an elementary Physical Education setting. Kinesiology, 44, 191-198.

Schmidt, R.A., \& Lee, T.D. (2011). Motor learning and control: A behavioral emphasis (5 ${ }^{\text {th }}$ ed.). Champaign, IL: Human Kinetics. 
Shea, J.B., \& Morgan, R.L. (1979). Contextual interference effects on the acquisition, retention, and transfer of a motor skill. Journal of Experimental Psychology: Human Learning and Memory, 5, 179-187.

Shea, C.H., \& Wright, D.L. (1995). Contextual dependencies: Influence on response latencies. Memory, 3, 81-95.

Smith, P.J.K. (2002). Task duration in contextual interference. Perceptual and Motor Skills, 95, 1155-1162.

Wulf, G., \& Lee, T.D. (1993). Contextual interference effects in movements of the same class: Differential effects on program and parameter learning. Journal of Motor Behavior, 25, 254-263.

Submitted: June 15, 2015

Accepted: May 9, 2016

Correspondence to:

Jared M. Porter

Department of Kinesiology

Southern Illinois University Carbondale

Mail Code: 4310

1075 South Normal Ave.

Carbondale, IL. 62901 USA

Phone: 1-618-453-3339

Fax: 1-618-453-3329

E-mail: jporter@siu.edu 